\title{
Vacinação Contra o Vírus do Papiloma Humano em Dermatologia
}

\author{
Ana Ortins-Pina ${ }^{1}$, Luís Soares-de-Almeida ${ }^{1,2,3}$, João Borges-Costa ${ }^{1,2,3}$ \\ ${ }^{1}$ Centro Hospitalar Lisboa Norte EPE, Hospital de Santa Maria, Serviço de Dermatologia. Lisboa, Portugal \\ ${ }^{2}$ Universidade de Lisboa, Faculdade de Medicina, Clínica Universitária de Dermatologia de Lisboa. Lisboa, Portugal \\ ${ }^{3}$ Instituto de Medicina Molecular. Lisboa, Portugal
}

RESUMO - A infeção anogenital pelo vírus do papiloma humano (VPH) é a infeção sexualmente transmissível (IST) mais frequente; devido ao seu potencial oncogénico, está na origem da maioria dos cancros do colo do útero, vulvar, vaginal e anal. Não existe tratamento antiviral específico e o tratamento das neoplasias associadas ao VPH não previne a transmissão. Assim, a prevenção da infeção assume particular relevância e a consulta de Venereologia constitui uma oportunidade privilegiada para aconselhar quem pode beneficiar de medidas preventivas. A mais recente vacina contra o VPH é a nonavalente, proporcionando cobertura para os tipos 6, 11, 16, 18, 31, 33, 45, 52 e 58, que, em conjunto, respondem por $90 \%$ dos cancros do colo do útero e condilomas. A vacina é recomendada pela OMS para mulheres e homens até aos 26 anos. Na maioria dos países, a vacina é comparticipada para proporcionar imunização universal gratuita a adolescentes do sexo feminino antes do início da atividade sexual. Para além da população-alvo, a vacina pode beneficiar outros populações-alvo frequentemente tratadas pelos dermatologistas: homens que têm sexo com homens, imunodeprimidos incluindo transplantados e infetados pelo $\mathrm{VIH}$, e candidatos a tratamentos imunossupressores. A decisão de vacinar um indivíduo deve considerar o risco de exposição prévia ao VPH e o benefício potencial da vacinação, que é profilática e não terapêutica. Revemos a evidência publicada sobre a imunogenicidade, segurança e eficácia da vacina contra o VPH em diferentes contextos clínicos e identificamos as recomendações internacionais que podem guiar o aconselhamento individual pelo dermatologista.

PALAVRAS-CHAVE - Condiloma Acuminado; Doenças da Pele Infecciosas; Hospedeiro Imunocomprometido; Infecções por Papillomavirus/prevenção e controlo; Vacinas contra Papillomavirus.

\section{Papillomavirus Vaccination in Dermatology}

ABSTRACT - Anogenital infection by human papillomavirus (HPV) is the most common sexually transmitted infection (STI). It bears oncogenic potential, causing most cases of cervical, vulvar, vaginal and anal cancer. There is no specific antiviral therapy and the treatment of HPV-associated neoplasms does not prevent transmission. Thus, prevention of infection is particularly important, and the Venereology consultation is a privileged opportunity to identify people who benefit from preventive measures. The most recent nonavalent HPV vaccine provides coverage for types 6, 11, 16, 18, 31, 33, 45, 52 and 58, which together account for $90 \%$ of cancers of the cervix and genital warts. The vaccine is recommended by the WHO for women and men up to 26 years old. In most countries, the vaccine is provided freely to ensure universal immunization of female adolescents prior to the commencement of sexual activity. In addition to the target population, the vaccine may benefit other individuals often treated by dermatologists: men who have sex with men, immunocompromised hosts such as organ-transplant and HIV-seropositive patients, and candidates for immunosuppressive treatments. The decision to vaccinate should consider the individual risk of prior exposure to HPV and the potential benefit of vaccination, which is prophylactic and not therapeutic. We review published data on the immunogenicity, safety and efficacy of the HPV vaccine in different clinical contexts, and international recommendations that may guide individual counseling by the dermatologist. KEYWORDS - Condylomata Acuminata; Immunocompromised Host; Papillomavirus Infections/prevention \& control; Papillomavirus Vaccines; Skin Diseases, Infectious.

Correspondência: Ana Ortins-Pina

Serviço de Dermatologia

Centro Hospitalar Lisboa Norte EPE - Hospital de Santa Maria

Avenida Prof. Egas Moniz

1649-035 Lisboa, Portugal

E-mail: ana.ortins@chln.min-saude.pt

DOI: https://dx.doi.org/10.29021/spdv.76.4.960
Recebido/Received

18 Junho/June 2018

Aceite/Accepted

17 Setembro/September 2018 


\section{INTRODUÇÃO}

A infeção anogenital pelo vírus do papiloma humano (VPH) é a infeção sexualmente transmissível (IST) mais frequente, assumindo especial relevância pelo seu potencial oncogénico. A prevalência da infeção por VPH varia entre $3 \%$ a $15 \%$ da população na Europa' e atinge $40 \%$ dos adultos dos 19 aos 59 anos de idade nos EUA. ${ }^{2,3} \mathrm{Em}$ Portugal, estima-se que a infeção por VPH afete $13 \%$ das mulheres entre os 18 e os 64 anos, sendo mais elevada entre os 20 e 24 anos (29\%). ${ }^{4}$ Para além de originar os condilomas, uma causa importante de morbilidade e frequente motivo de consulta, a infeção anogenital por VPH responde pela maioria dos casos de cancro do colo do útero, vulvar, vaginal, anal e orofaríngeo. ${ }^{5} \mathrm{O}$ cancro do colo do útero causa aproximadamente 275000 mortes/ ano no mundo. ${ }^{5}$

Não existe tratamento antiviral específico, pelo que a prevenção da infeção assume particular relevância. Sendo o dermatologista frequentemente responsável por diagnosticar a doença associada à infeção por VPH, bem como outras IST, a consulta de Dermatologia e Venereologia constitui uma oportunidade privilegiada para aconselhar os doentes que beneficiam de medidas preventivas contra a infeção por VPH. Aprovada em 2014 pela Food and Drug Administration (FDA) para mulheres e homens até aos 26 anos, a Gardasil9 ${ }^{\circledR}$ é a mais recente vacina profilática contra o VPH. Revemos a evidência publicada sobre a imunogenicidade, segurança e eficácia da vacina contra o VPH em diferentes contextos clínicos e identificamos as recomendações internacionais que podem guiar o aconselhamento individual pelo dermatologista.

\section{MÉTODOS}

Foi realizada uma pesquisa bibliográfica na PubMed, com os termos «human papillomavirus vaccine», «anogenital warts», «immunocompromised host», "cervical cancer», «HPV associated cancer», "cervical screening», "guidelines», em diferentes combinações. Foram incluídos artigos em inglês e em português, publicados nos últimos 20 anos, i.e. posteriormente a 1997. Foram seleccionados os artigos originais pertinentes sobre a segurança e eficácia da vacina, os artigos de revisão e recomendações com maior relevância para a Dermatologia, e foram também revistas as suas referências bibliográficas.

\section{RESULTADOS}

A vacina Gardasil9 ${ }^{\circledR}$ contém, como imunogénio, partículas virais da capside L1. Não contendo DNA, não tem potencial infecioso ou replicativo. Na sua forma mais recente, a Gardasil9 ${ }^{\circledR}$ é nonavalente, em contraste com a sua predecessora (Gardasil ${ }^{\circledR}$ tetravalente). Para além dos tipos 6, 11, 16 e 18, proporciona cobertura para 031 , $33,45,52$ e 58 . Em conjunto, os tipos 16, 18, 31, 33, 45, 52,58 são responsáveis por $90 \%$ dos casos de cancro do colo do útero. ${ }^{6}$
A Gardasil9 ${ }^{\circledR}$, de acordo com o RCM, está aprovada para a imunização ativa de indivíduos a partir dos 9 anos de idade, para a prevenção das doenças causadas pelos tipos de VPH da vacina, incluindo lesões pré-cancerosas e cancro do colo do útero, vulva, vagina e ânus, e condilomas. Deve ser administrada por via subcutânea em esquema de duas doses (0, 6 meses) e, nos indivíduos com 15 anos ou mais ou imunocomprometidos, em esquema de três doses $(0,2,6$ meses), no intervalo máximo de 1 ano. Como parte do Plano Nacional de Vacinação (PNV) desde 2008, a vacina profilática contra o VPH atingiu uma cobertura de $85 \%$ na população-alvo, as raparigas entre 9 e 13 anos de idade. ${ }^{7}$ A vacina é recomendada aos 10 anos e, desde 2017, a Gardasil9 ${ }^{\circledR}$ nonavalente substituiu a tetravalente.

A vacina é segura, sendo os efeitos secundários mais frequentes as reações locais (84\%) e as cefaleias (13\%), que podem ocorrer, respetivamente, nos primeiros 5 e 15 dias posteriormente à administração. ${ }^{8}$ Tal como com outras vacinas, pode ocorrer síncope, o que implica um período de vigilância de 30 a 60 minutos após a administração. Podem também ocorrer tonturas, náuseas, febre e astenia (>1 em 100 vacinados). ${ }^{8}$ A vacina não contém timerosal nem outros conservantes, mas está contra-indicada em casos de hipersensibilidade a algum dos componentes: sulfato de hidroxifosfato de alumínio, L-histidina, polissorbato 80 , borato de sódio.

A eficácia da vacina está demonstrada em estudos clínicos e epidemiológicos. Nas mulheres até aos 26 anos de idade, a vacina previne $100 \%$ dos condilomas, neoplasia intraepitelial vaginal, vulvar e cervical. ${ }^{9}$ Nos homens, previne $90 \%$ dos condilomas e neoplasia intraepitelial anal. ${ }^{10}$ Com uma taxa de seroconversão de $99 \%$, a resposta imunitária persiste por, pelo menos, 5 anos e a proteção pode durar 8 anos após a administração. ${ }^{9}$ Os primeiros dados epidemiológicos foram obtidos na Austrália, nos períodos de 2003 a 2006 e de 2007 a 2010 e com uma cobertura vacinal de $70 \%$ das raparigas com 14 a 19 anos, mostraram uma redução da prevalência de infeção cervical por VPH de 11,5\% para $5 \%$ e o quase desaparecimento de condilomas em ambos os sexos antes dos 21 anos. ${ }^{11}$ Perante a eficácia da vacina, é expectável que as taxas de positividade nas citologias cervicovaginais de rastreio venham a diminuir. ${ }^{12}$ Evidência recente sugere que o rastreio entre as mulheres vacinadas possa ser mais simples, com menor número de visitas, sem prejuízo da efetividade e com melhor adesão, pelo que alguns autores defendem a atualização das recomendações nacionais. ${ }^{13}$ Relativamente ao carcinoma orofaríngeo, que em $70 \%$ dos casos é positivo para $\mathrm{VPH}, 9$ ainda não existem dados de eficácia ou epidemiológicos, mas alguns autores consideram possível que a Gardasil ${ }^{\circledR}$ venha a reduzir a sua incidência. ${ }^{14}$

Apesar de relatos casuísticos de doentes cujas lesões regrediram após a vacinação com Gardasi ${ }^{\circledR}$, 15,16 não há 
evidência que suporte o uso terapêutico da vacina contra doença estabelecida. Pelo contrário, a imunidade celular, mais do que a humoral, parece ser necessária para a remissão das neoplasias associadas ao $\mathrm{VPH} .{ }^{17}$

O esquema vacinal na maioria dos países tem como objetivo a imunização universal e gratuita da população-alvo primária: adolescentes do sexo feminino antes do início da atividade sexual. A vacinação de populações-alvo secundárias - mulheres dos 15 aos 26 anos e homens - é menos custo-eficaz, sendo recomendada pela OMS se for financeiramente viável. ${ }^{18}$ Os indivíduos do sexo masculino não são vacinados em muitos países, esperando-se o efeito da imunidade de grupo obtida pela vacinação das raparigas. As limitações da cobertura não universal são evidentes nos homens que têm sexo com homens $(\mathrm{HSH})$, onde a prevalência da infeção anal por VPH atinge $75 \% .{ }^{19}$ Alguns países - Canadá, EUA, Áustria e, mais recentemente, Reino Unido - englobam todos os adolescentes no programa nacional de vacinação independentemente do género.

A eficácia clínica da vacina é superior em mulheres sem evidência de infeção prévia por VPH, confirmando a importância da vacinação antes do início da atividade sexual. Contudo, há um aumento do risco oncológico entre os doentes diagnosticados com condilomas, ${ }^{20}$ que pode ser atribuído a diferentes fatores: maior exposição a coinfeções por tipos oncogénicos de VPH, maior susceptibilidade individual para a persistência da infeção por VPH, fatores de risco comuns a condilomas e outras neoplasias, como imunossupressão e tabagismo, e promoção da oncogénese pela inflamação anogenital secundária à infeção por VPH.

Assim, desde cedo se propõe vacinar também as muIheres com história pessoal de condilomas com o objetivo de as proteger contra os outros subtipos de $\mathrm{VPH}^{21}$ e, na prática, qualquer adulto sexualmente ativo pode beneficiar de proteção contra a infeção por VPH de tipos aos quais não tenha sido exposto previamente. Contudo, a imunogenicidade diminui com a idade da vacinação. Com uma taxa de seroconversão semelhante, os níveis de anticorpos são inferiores nos indivíduos com mais de 15 anos. ${ }^{22}$ Contudo, a relevância clínica desta observação pode ser diminuta, considerando que a Gardasil ${ }^{\circledR}$, em mulheres dos 24 aos 45 anos, teve uma eficácia combinada de $89 \%$ na prevenção de infeção por VPH e condilomas, neoplasia vaginal e cancro do colo do útero. $\mathrm{Nos} \mathrm{HSH}$, foi demonstrada a redução em $75 \%$ da incidência de neoplasia intraepitelial anal após a vacinação. ${ }^{23}$

Nos doentes imunodeprimidos, a infeção por VPH é mais frequente, mais grave e mais refratária aos tratamentos. ${ }^{24}$ Por exemplo, mulheres infetadas pelo $\mathrm{VIH}$ têm uma prevalência 2 a 3 vezes superior de infeção por $\mathrm{VPH}^{25}$ e um risco de cancro do colo do útero 2 a 22 vezes superior. ${ }^{26} \mathrm{O}$ risco de displasia intraepitelial anal de alto grau nos $\mathrm{HSH}$ com infeção VIH é $49 \%$, comparativamente a um risco de $17 \%$ em HSH sem infeção VIH. ${ }^{27}$ Em contraste com outras infeções virais em doentes VIH-seropositivos, o tratamento antirretroviral não parece impedir a transmissão e progressão da doença provocada pelo $\mathrm{VPH} .^{28}$

Relativamente à segurança nos doentes imunodeprimidos, não se identificaram efeitos adversos significativos da vacina contra o VPH em doentes com infeção $\mathrm{VIH}$, doenças autoimunes e transplantados. ${ }^{29}$ Nomeadamente, a vacinação contra VPH não aumentou o risco de flare em doentes com lúpus eritematoso ${ }^{30,31}$ e não teve efeitos adversos na contagem de células CD4 ou carga viral em doentes com infeção $\mathrm{VIH} .{ }^{32} \mathrm{Em}$ doentes $\mathrm{VIH}$-seropositivos, as taxas de seroconversão obtidas com a vacina contra o VPH foram superiores a $95 \%,{ }^{33}$ incluindo doentes com e sem tratamento antirretroviral. Contudo, a imunogenicidade da vacina é menor em doentes iatrogenicamente imunossuprimidos (após transplante, ${ }^{29}$ doenças inflamatórias autoimunes, ${ }^{31} \mathrm{e}$ em doentes sob terapêuticas biológicas ${ }^{34}$ ), com taxas de seroconversão e níveis de anticorpos inferiores aos observados em imunocompetentes. A imunogenicidade difere de acordo com o tipo e grau de imunossupressão. São ineficazes as vacinas administradas nos primeiros 3 a 6 meses após transplante de órgão-sólido ou de células hematopoiéticas, e nos 6 meses após terapêutica com rituximab. ${ }^{35} \mathrm{~A}$ redução na imunogenicidade da vacina parece ser maior com micofenolato de mofetil, e diretamente relacionada com a concentração sérica de tacrolimus. Nos doentes transplantados, observam-se taxas de seroconversão entre 53\% - 68\%, ${ }^{29}$ sendo tanto menor quanto mais precoce for a vacinação após o transplante. Em doentes com lúpus eritematoso sistémico, a taxa de seroconversão após a vacina contra - VPH é $76 \%$ - 95\%. ${ }^{31}$ São escassos os estudos sobre a eficácia da vacina na redução da incidência, gravidade e morbimortalidade da infeção por VPH nos doentes imunocomprometidos, sendo expectável que, tal como nos imunocompetentes, a vacina seja tanto menos eficaz quanto maior for a idade e o grau de exposição prévia. Por exemplo, doentes VIH-positivos com mais de 26 anos não parecem beneficiar de proteção significativa contra infeção anal persistente. ${ }^{32}$

A redução de imunogenicidade da vacina em doentes imunodeprimidos não implica necessariamente ausência de benefício clínico, e a escassez de evidência não significa falta de eficácia. Assim, tendo em conta a incidência e gravidade da infeção por VPH em doentes imunocomprometidos, e sendo a vacina segura nestes doentes, várias sociedades internacionais recomendam a vacina contra o $\mathrm{VPH}$ em doentes imunocomprometidos ${ }^{36}$ e em candidatos a imunossupressão, antes de iniciar o tratamento, com 3 doses da vacina. ${ }^{37}$ Segundo o Advisory comittee for immunization panel do CDC, Infectious Diseases Society of America, e International Papillomavirus Society, em geral, devem ser vacinados os doentes com infeção $\mathrm{VIH}$ e todos os candidatos a tratamento imunossupressor (Tabela 1). As recomendações de vacinação contra o VPH devem ser 


\section{GEIDST}

Tabela 1 - Recomendações relativas à vacina contra o VPH, relevantes para a Dermatologia e Venereologia.

\begin{tabular}{|c|c|c|c|}
\hline PNV & 우 10 anos & - & - \\
\hline ACIP/CDC $(2015)^{38}$ & $\begin{array}{l}\text { - } q \leq 26 \text { anos* } \\
\text { - } 011-21 \text { anos (pode } \\
\text { alargar até } 26 \text { anos). } \\
\text { - } \lesssim \mathrm{HSH} \leq 26 \text { anos }\end{array}$ & $\begin{array}{l}\text { Doentes imunocomprometidos, } \\
\text { incluindo VIH-positivos, } \leq 26 \text { anos. }\end{array}$ & - \\
\hline IDSA $(2013)^{35}$ & & $\begin{array}{l}\text { - Todos os doentes VIH-positivos dos } \\
11 \text { aos } 26 \text { anos (recomendação } \\
\text { forte). } \\
\text { - Não recomendada durante quimiote } \\
\text { rapia e nos } 3 \text { meses seguintes. }\end{array}$ & $\begin{array}{l}\text { - } 11-26 \text { anos, antes de transplante de } \\
\text { órgão sólido (ou depois). } \\
\text { - Vacinação possível } \geq 2 \text { semanas antes } \\
\text { de iniciar imunossupressão }\end{array}$ \\
\hline $\begin{array}{l}\text { National Psoriasis } \\
\text { Foundation }(2013)^{39}\end{array}$ & & & $\begin{array}{l}\text { - Todos os doentes com psoríase } \leq 26 \\
\text { anos, candidatos a tratamentos biológi- } \\
\text { cos (antes ou durante o tratamento). }\end{array}$ \\
\hline
\end{tabular}

* (*) Os dados de ensaios clínicos demonstram a eficácia da vacina em mulheres até aos 45 anos de idade. (£) As normas da IDSA são omissas quanto à vacinação profilática do VPH especificamente em doentes reumatológicos/com doenças inflamatórias crónicas. (§) É encorajada a cobertura vacinal do maior número de tipos de VPH possível nos doentes infetados pelo VIH, uma vez que nestes, há uma distribuição mais alargada de tipos de VPH nas neoplasias associadas. $\mathrm{e}$ e : homens e mulheres, respectivamente. ACIP, Advisory Comittee for Immunization Panel; CDC, Center for Disease Control; HSH, homens que têm sexo com homens; IDSA, Infectious Diseases Society of America; IPVS, International Papillomavirus Society; OMS, Organização Mundial de Saúde; PNV, Plano Nacional de Vacinação; RCM, resumo das características do medicamento; VIH, vírus da imunodeficiência humana; VPH, vírus do papiloma humano.

adaptadas a cada doente, tendo em conta o género, a idade (especialmente recomendada até aos 26 anos), patologia de base, tipo e intensidade de imunossupressão.

\section{CONCLUSÃO}

O dermatologista tem um papel central na prevenção da infeção por VPH e morbilidade associada. A decisão de vacinar depende da avaliação, caso-a-caso, com base na evidência publicada e recomendações existentes. $\mathrm{O}$ benefício da vacina depende da exposição prévia ao VPH, probabilidade de adquirir a infeção, risco de complicações associadas e imunogenicidade, tendo em conta a idade e comorbilidades. Não sendo comparticipada pelo SNS, a vacina tem um custo que pode não ser acessível a todos os doentes.

É importante sublinhar que a vacinação não é $100 \%$ eficaz, não previne a infeção por todos os tipos de VPH e não tem eficácia terapêutica documentada. Assim, a vacina contra o $\mathrm{VPH}$ não substitui nem deve atenuar as precauções contra a transmissão de IST e não dispensa as medidas recomendadas para rastreio do cancro do colo do útero, as quais poderão, contudo, vir a ser atualizadas.
Conflitos de interesse: Os autores declaram não possuir conflitos de interesse.

Suporte financeiro: $O$ presente trabalho não foi suportado por nenhum subsídio ou bolsa.

Conflicts of interest: The authors have no conflicts of interest to declare.

Financing Support: This work has not received any contribution, grant or scholarship.

\section{REFERÊNCIAS}

1. De Vuyst H, Clifford G, Li N, Franceschi S. HPV infection in Europe. Eur J Cancer. 2009; 45:2632-9.

2. Hariri S, Unger E, Sternberg M, Dunne E, Swan D, Patel $S$, et al. Prevalence of genital human papillomavirus among females in the United States, the National Health and Nutrition Examination Survey, 2003-2006. J Infect Dis. $2011 ; 204: 566-73$. doi: 10.1093/infdis/jir341.

3. Gargano J, Unger E, Liu G, Steinau M, Meites E, Dunne $E$, et al. Prevalence of Genital Human Papillomavirus in Males, United States, 2013-2014. J Infect Dis. 2017; 215:1070-9. doi: 10.1093/infdis/jix057. 
4. Pista A, de Oliveira C, Cunha M, Paixão T, Real O. CLEOPATRE Portugal Study Group. Prevalence of Human Papillomavirus Infection in Women in Portugal: The CLEOPATRE Portugal Study. Int J Gynecol Cancer. 2011 ; 21:1150-8. doi: 10.1097/IGC.0b013e31821dd3b2.

5. Schiller JT, Lowy DR. Understanding and learning from the success of prophylactic human papillomavirus vaccines. Nat Rev Microbiol. 2012;10:681-92. doi: 10.1038/ nrmicro2872.

6. Li N, Franceschi S, Howell-Jones R, Snijders P, Clifford G. Human papillomavirus type distribution in 30,848 invasive cervical cancers worldwide: variation by geographical region, histological type and year of publication. Int J Cancer. 2011 ; 128:927-35. doi: 10.1002/ijc. 25396.

7. Direção Geral Da Saúde. Programa Nacional de Vacinação 2017. Lisboa: DGS; 2016.

8. Moreira EDJ, Block SL, Ferris D, Giuliano AR, Iversen O-E, Joura EA, et al. Safety Profile of the 9-Valent HPV Vaccine: A Combined Analysis of 7 Phase III Clinical Trials. Pediatrics. 2016;138:e 20154387. doi: 10.1542/ peds.2015-4387.

9. Markowitz LE, Dunne EF, Saraiya M, Chesson HW, Curtis CR, Gee J, et al. Human papillomavirus vaccination: recommendations of the Advisory Committee on Immunization Practices (ACIP). Morb Mortal Wkly report Recomm reports. 2014;63:1-30.

10. Giuliano A, Palefsky J, Goldstone S, Moreira ED, Penny $M E$, Aranda $C$, et al. Efficacy of quadrivalent HPV vaccine against HPV Infection and disease in males. N Engl J Med. 2011;364:401-11. doi: 10.1056/NEJMoa0909537.

11. Read T, Hocking J, Chen M, Donovan B, Bradshaw C, Fairley $C$. The near disappearance of genital warts in young women 4 years after commencing a national human papillomavirus (HPV) vaccination programme. Sex Transm Infect. $2011 ; 87: 544-7$. doi: 10.1136/sextrans-2011-050234.

12. Hestbech MS, Lynge E, Kragstrup J, Siersma V, Baillet $M V$, Brodersen J. The impact of HPV vaccination on future cervical screening: A simulation study of two birth cohorts in Denmark. BMJ Open. 2015;5:1-7. doi: 10.1136/bmjopen-2015-007921.

13. Landy R, Windridge P, Gillman MS, Sasieni PD. What cervical screening is appropriate for women who have been vaccinated against high risk HPV? A simulation study. Int J Cancer. 2018; 142:709-18. doi: 10.1002/ijc.31094.

14. Herrero R, Quint W, Hildesheim A, P G, Struijk L, Katki $H$, et al. Reduced prevalence of oral human papillomavirus (HPV) 4 years after bivalent HPV vaccination in a randomized clinical trial in Costa Rica. PLoS One. 2013;8:e68329. doi: 10.1371/journal.pone.0068329.

15. Silling S, Wieland U, Werner M, Pfister H, Potthoff A, Kreuter A. Resolution of novel human papillomavirus-induced warts after HPV vaccination. Emerg Infect Dis. 2014; 20:142-5. doi: 10.3201/eid2001.130999.

16. Smith SP, Baxendale HE, Sterling JC. Clearance of recalcitrant warts in a patient with idiopathic immune deficiency following administration of the quadrivalent human papillomavirus vaccine. Clin Exp Dermatol. 2017; 42:306-8. doi: 10.1111/ced.13038.

17. Howley P, Lowy D. Papillomaviruses. In: Knipe D, Howley P, editors. Fields virology. 5th ed. Philadelphia: Lippincott Williams \& Wilkins; 2011 . p. 2299-354.

18. World Health Organization. Human papillomavirus vaccines: WHO position paper, May 2017. Wkly Epidemiol Rec. 2017;92:241-68.

19. Stanley M. HPV vaccination in boys and men. Hum Vaccin Immunother. 2014;10:2109-11.

20. Vaz N, Borges-Costa J, Marques MS. Condilomas genitais e associação com cancro. Rev Soc Port Dermatol Venereol. 2013;71:59-63.

21. Leite I, Lisboa C, Azevedo F. Vírus do Papiloma Humano e Vacinação. Rev Soc Port Dermatol Venereol. 2011 ; 69:395-402.

22. Hillman R, Giuliano A, Palefsky J, Goldstone S, Moreira $E$, Vardas $E$, et al. Immunogenicity of the quadrivalent human papillomavirus (type 6/11/16/18) vaccine in males 16 to 26 years old. Clin Vaccine Immunol. 2012; 19:261-7. doi: 10.1128/CVI.05208-11.

23. Palefsky J, Giuliano A, Goldstone S, Moreira E, Aranda $C$, Jessen $H$, et al. HPV Vaccine against anal HPV infection and anal intraepithelial neoplasia. N Engl J Med. 2011 ; 365:1576-85. doi: 10.1056/NEJMoa1010971.

24. Reusser N, Downing C, Guidry J, Tyring S. HPV Carcinomas in immunocompromised patients. J Clin Med. 2015; 4:260-81. doi: 10.3390/jcm4020260.

25. Jamieson DJ, Duerr A, Burk R, Klein RS, Paramsothy $P$, Schuman $P$, et al. Group HERS Characterization of genital human papillomavirus infection in women who have or who are at risk of having HIV infection. Am J Obstet Gynecol. 2002; 186:21-7.

26. Vuyst H De, Lillo F, Broutet N, Smith JS. HIV, human papillomavirus, and cervical neoplasia and cancer in the era of highly active antiretroviral therapy. Eur J Cancer Prev. 2008;17:545-54. doi: 10.1097/ CEJ.0b013e3282f75ea1.

27. Palefsky JM, Holly E, Ralston M, Jay N, Berry J, Darragh T. High incidence of anal high-grade squamous intra-epithelial lesions among HIV-positive and HIV-negative homosexual and bisexual men. AIDS. 1998; 12:495503.

28. Frisch M, Biggar R, Goedert J. Human papillomavirus-associated cancers in patients with human immunodeficiency virus infection and acquired immunodeficiency syndrome. J Natl Cancer Inst. 2000; 92:1500-10.

29. Kumar D, Unger ER, Panicker G, Medvedev P, Wilson $L$, Humar A, et al. Immunogenicity of quadrivalent human papillomavirus vaccine in organ transplant recipients. Am J Transpl. 2013;13:2411-7. doi: 10.1111/ ajt. 12329.

30. Dhar JP, Essenmacher L, Dhar R, Magee A, Ager J, Sokol RJ. The safety and immunogenicity of Quadrivalent HPV 
(qHPV) vaccine in systemic lupus erythematosus. Vaccine. 2017;35:2642-6. doi: 10.1016/i.vaccine.2017.04.001

31. Mok CC, Ho LY, Fong LS, To CH. Immunogenicity and safety of a quadrivalent human papillomavirus vaccine in patients with systemic lupus erythematosus: a case-control study. Ann Rheum Dis. 2013;72:659-64. doi: 10.1136/annrheumdis-2012-201393.

32. Wilkin TJ, Chen H, Cespedes MS, Leon-Cruz JT, Godfrey C, Chiao EY, et al. A randomized, placebo-controlled trial of the quadrivalent HPV vaccine in HIV-infected adults age 27 years or older: AIDS Clinical Trials Group protocol A5298 [Internet]. Clin Infect Dis. 2018 (in press). doi: 10.1093/cid/ciy274.

33. Levin MJ, Moscicki A, Song L, Fenton T, Nowak B, Sattler $\mathrm{C}$ a, et al. Safety and immunogenicity of a quadrivalent human papillomaviru (types 6, 11, 16, and 18) vaccine in HIV-infected children 7 to 12 years old. J Acquir Immune Defic Syndr. 2010;55:197-204. doi: 10.1097/ QAI.0b013e3181de8d26.

34. Lebwohl M, Bagel J, Gelfand J, Gladman D, Gordon K, Hsu S, et al. From the Medical Board of the National Psoriasis Foundation: monitoring and vaccinations in patients treated with biologics for psoriasis. J Am Acad Dermatol. 2008;58(1):94-105.
35. Rubin LG, Levin MJ, Ljungman P, Davies EG, Avery R, Tomblyn M, et al. 2013 IDSA Clinical Practice Guideline for Vaccination of the Immunocompromised Host. Clin Infect Dis. 2014;58:e44-100. doi: 10.1093/cid/cit684.

36. Lopez A, Mariette X, Bachelez H, Belot A, Bonnotte B, Hachulla $E$, et al. Vaccination recommendations for the adult immunosuppressed patient: A systematic review and comprehensive field synopsis. J Autoimmun. 2017; 80:10-27. doi: 10.1016/i.jaut.2017.03.011.

37. Garland SM, Brotherton JML, Moscicki AB, Kaufmann AM, Stanley M, Bhatla N, et al. HPV vaccination of immunocompromised hosts. Papillomavirus Res. 2017; 4:35-8.

38. Emiko Petrosky, Joseph A. Bocchini, Susan Hariri, Harrell Chesson, C. Robinette Curtis, Mona Saraiya, MD5, Elizabeth R. Unger LEM. Use of 9-Valent Human Papillomavirus (HPV) Vaccine: Updated HPV Vaccination Recommendations of the Advisory Committee on Immunization Practices. MMWR Morb Mortal Wkly Rep. 2015; 64:300-4.

39. Wine-Lee L, Keller SC, Wilck MB, Gluckman SJ, Van Voorhees AS. From the Medical Board of the National Psoriasis Foundation: Vaccination in adult patients on systemic therapy for psoriasis. J Am Acad Dermatol. 2013; 69:1003-13. doi: 10.1016/i.jaad.2013.06.046. 